\title{
Non-Laboratory-Based Simple Screening Model for Nonalcoholic Fatty Liver Disease in Patients with Type 2 Diabetes Developed Using Multi-Center Cohorts
}

\begin{abstract}
Jiwon Kim ${ }^{1, *}$, Minyoung Lee ${ }^{1, *}$, Soo Yeon Kim² ${ }^{2}$ i-Hye Kim³ ${ }^{3}$ Ji Sun Nam², Sung Wan Chun ${ }^{5}$, Se Eun Park ${ }^{6}$, Kwang Joon Kim ${ }^{1}$, Yong-ho Lee ${ }^{1}$, Joo Young Nam${ }^{7}$, Eun Seok Kang ${ }^{1}$

${ }^{1}$ Department of Internal Medicine, Severance Hospital, Yonsei University College of Medicine; ${ }^{2}$ Department of Education and Training, Severance Hospital, Yonsei University College of Medicine; ${ }^{3}$ Severance Health Check-up, Severance Hospital, Yonsei University Health System; ${ }^{4}$ Division of Endocrinology and Metabolism, Department of Internal Medicine, Gangnam Severance Hospital, Yonsei University College of Medicine, Seoul; ${ }^{5}$ Division of Endocrinology and Metabolism, Department of Internal Medicine, Soonchunhyang University Cheonan Hospital, Cheonan; ${ }^{6}$ Division of Endocrinology and Metabolism, Department of Internal Medicine, Kangbuk Samsung Hospital, Sungkyunkwan University School of Medicine, Seoul; ${ }^{7}$ Division of Endocrinology and Metabolism, Department of Internal Medicine, National Health Insurance Service Ilsan Hospital, Goyang, Korea
\end{abstract}

Background: Nonalcoholic fatty liver disease (NAFLD) is the most prevalent cause of chronic liver disease worldwide. Type 2 diabetes mellitus (T2DM) is a risk factor that accelerates NAFLD progression, leading to fibrosis and cirrhosis. Thus, here we aimed to develop a simple model to predict the presence of NAFLD based on clinical parameters of patients with T2DM.

Methods: A total of 698 patients with T2DM who visited five medical centers were included. NAFLD was evaluated using transient elastography. Univariate logistic regression analyses were performed to identify potential contributors to NAFLD, followed by multivariable logistic regression analyses to create the final prediction model for NAFLD.

Results: Two NAFLD prediction models were developed, with and without serum biomarker use. The non-laboratory model comprised six variables: age, sex, waist circumference, body mass index (BMI), dyslipidemia, and smoking status. For a cutoff value of $\geq 60$, the prediction accuracy was 0.780 ( $95 \%$ confidence interval [CI], 0.743 to 0.817 ). The second comprehensive model showed an improved discrimination ability of up to 0.815 ( $95 \%$ CI, 0.782 to 0.847 ) and comprised seven variables: age, sex, waist circumference, BMI, glycated hemoglobin, triglyceride, and alanine aminotransferase to aspartate aminotransferase ratio. Our non-laboratory model showed non-inferiority in the prediction of NAFLD versus previously established models, including serum parameters. Conclusion: The new models are simple and user-friendly screening methods that can identify individuals with T2DM who are at

Received: 17 April 2021, Revised: 28 June 2021, Accepted: 16 July 2021

Corresponding authors: Eun Seok Kang

Division of Endocrinology and Metabolism, Department of Internal Medicine, Yonsei University College of Medicine, 50-1 Yonsei-ro, Seodaemun-gu, Seoul 03722 , Korea

Tel: +82-2-2228-1968, Fax: +82-2-393-6884, E-mail: edgo@yuhs.ac

Joo Young Nam

Division of Endocrinology and Metabolism, Department of Internal Medicine, National Health Insurance Service Ilsan Hospital, 100 Ilsan-ro, Ilsandong-gu, Goyang 10444, Korea

Tel: +82-31-900-0340, Fax: +82-31-900-0343, E-mail: njy0525@nhimc.or.kr
Copyright $\odot 2021$ Korean Endocrine Society

This is an Open Access article distributed under the terms of the Creative Commons Attribution Non-Commercial License (https://creativecommons.org/ licenses/by-nc/4.0/) which permits unrestricted non-commercial use, distribution, and reproduction in any medium, provided the original work is properly cited.

*These authors contributed equally to this work. 
high-risk for NAFLD. Additional studies are warranted to validate these new models as useful predictive tools for NAFLD in clinical practice.

Keywords: Non-alcoholic fatty liver disease; Diabetes mellitus, type 2; Transient elastography; Screening

\section{INTRODUCTION}

The prevalence of nonalcoholic fatty liver disease (NAFLD) is increasing worldwide, making it one of the most common causes of chronic liver disease [1]. NAFLD is defined as an excessive hepatic fat accumulation of more than 5\% in hepatocytes without secondary causes such as significant alcohol intake, steatogenic medications, or viral hepatitis [2]. NAFLD, a heterogeneous disease that covers a wide spectrum of presentations from simple steatosis to nonalcoholic steatohepatitis (NASH), is associated with hepatocyte ballooning that can progress to cirrhosis and even hepatocellular carcinoma [2,3]. The clinical importance of NAFLD includes its relationship with advanced liver diseases and its close association with insulin resistance, type 2 diabetes mellitus (T2DM), obesity, and metabolic syndrome, leading to an increased risk of cardiovascular morbidity $[3,4]$.

T2DM is associated with a worse prognosis of NAFLD. In T2DM, the estimated prevalence of NAFLD is 55.5\%, approximately two-fold higher than that in the general population [5]. Among patients with T2DM and concomitant NAFLD, 37.3\% have NASH, an advanced form of NAFLD [5]. In addition, T2DM accelerates NAFLD progression two-fold compared to NAFLD alone [6]. Meanwhile, accumulating evidence shows that NAFLD aggravates microvascular and macrovascular complications in T2DM $[4,7,8]$. Thus, the early detection and intervention of NAFLD are especially important for patients with T2DM to reduce morbidity and mortality rates.

Although liver biopsy is the gold standard for assessing NAFLD [4], its invasive nature and sampling variability limit its routine use. Furthermore, imaging modalities for the diagnosis of NAFLD, such as hepatic ultrasound, computed tomography (CT), or magnetic resonance imaging (MRI), are expensive, inconvenient, and sometimes unavailable in community hospital-based settings. Therefore, for the timely diagnosis of NAFLD, clinical prediction models using various laboratory parameters have been developed to screen high-risk patients. For example, alanine aminotransferase (ALT) and aspartate aminotransferase (AST) are the most commonly used laboratory parameters in clinical prediction models of NAFLD [4,9]. However, these models require hospital visits with additional blood sampling for the prediction of NAFLD and cannot be easily applied to patients. Although a non-laboratory-based scoring model for NAFLD was recently suggested [10], it has not been validated in patients with T2DM alone.

Thus, we investigated the clinical risk factors associated with NAFLD in patients with T2DM and developed a non-laboratory parameter-based simple screening model for NAFLD in these patients. We also attempted to provide a more comprehensive model with superior predictive power for NAFLD using laboratory parameters in patients with T2DM. These prediction models were developed and validated using a combined health screening database from five medical centers and liver transient elastography (TE) to determine the presence and degree of NAFLD.

\section{METHODS}

\section{Subjects}

This cross-sectional study collected data from five medical centers (four tertiary hospitals, one secondary hospital) in Korea between January 2005 and August 2018: Severance Health Check-up Center, Gangnam Severance Hospital, Soonchunhyang University Cheonan Hospital, Kangbuk Samsung Hospital, and National Health Insurance Service Ilsan Hospital. This study included a total of 847 subjects who were diagnosed with T2DM and simultaneously evaluated using TE. Patients were defined as having T2DM if they met at least one of the following criteria: (1) use of oral hypoglycemic agent or insulin; (2) previous diagnosis by a physician; (3) glycated hemoglobin (HbA1c) level of $\geq 6.5 \%$; and (4) fasting plasma glucose concentration of $\geq 126 \mathrm{mg} / \mathrm{dL}$ [11]. The presence of NAFLD was defined as a controlled attenuation parameter (CAP) of $\geq 238$ $\mathrm{dB} / \mathrm{m}$ or steatosis stage 1 (S1) on TE [12]. Of the 847 patients, 149 were excluded based on the following exclusion criteria: (1) excessive alcohol consumption $(\geq 210 \mathrm{~g} /$ week for males and $\geq 140 \mathrm{~g} /$ week for females) ( $n=94$ ) [3]; (2) hepatitis B or hepatitis $\mathrm{C}$ infection $(n=37)$; (3) increased total and direct bilirubin 
concentrations $(n=16)$; and (4) missing anthropometric measurements $(n=2)$. A total of 698 participants with T2DM were enrolled in the final analysis.

The study protocol was approved by the Institutional Review Board of the Yonsei University College of Medicine, Seoul, Republic of Korea (approval no. 4-2018-0927).

\section{Laboratory assessment and data collection}

Each patient underwent anthropometry, laboratory tests, TE, and questionnaire assessments. We obtained demographic and anthropometric data including age, sex, height, weight, and waist circumference and calculated body mass index (BMI) by dividing the body weight by the height squared $\left(\mathrm{kg} / \mathrm{m}^{2}\right)$. Blood pressure was measured using standard methods, and data concerning personal medical history, current medication, and habitual lifestyle factors, such as alcohol consumption, smoking status, and physical activity, were collected via questionnaires. Blood samples were collected the morning after an 8-hour overnight fast. Hypertension was defined as the current use of antihypertensive medications or previous diagnosis by a clinical physician. We defined dyslipidemia as the use of a cholesterollowering agent at the time of study enrollment or previous diagnosis by a health care professional. Alcohol intake was self-reported by questionnaires inquiring about the frequency of drinking, amount of alcohol consumed per drinking event, and types of beverages and quantified by calculating the daily alcohol consumption [10]. After excluding heavy drinkers according to the exclusion criteria, we categorized drinking status as nondrinkers or current drinkers. Current drinker is defined as a person who drinks at least once a week. Smoking status was categorized according to lifelong cigarette exposure as current smokers or non-current smokers, which included never smokers and past smokers. Physical activity was also self-reported, including exercise frequency, duration, and intensity. Regular exercisers were defined as those patients who exercised for at least 30 minutes twice per week or more.

\section{Measurements of hepatic steatosis and fibrosis by TE}

TE was recently accepted as a noninvasive and reliable tool for measuring liver steatosis and fibrosis. The CAP, a novel method for assessing hepatic steatosis, is based on the ultrasonic properties of attenuation obtained at $3.5 \mathrm{MHz}$ using a FibroScan ${ }^{\circledR}$ device (Echosens, Paris, France) [12]. The basic principle of this noninvasive medical tool based on ultrasound attenuation has been described previously [12]. In addition, Fraquelli et al. [13] reported an excellent reproducibility of $\mathrm{TE}$, and the intraclass correlation coefficients of both intraobserver and interobserver agreement were 0.98 for assessing hepatic fibrosis. TE was performed by well-trained technicians who were blinded to the patients' clinical and biochemical information. Regular acquisition procedures were performed as previously described with the patient lying in the dorsal decubitus position with the right arm in maximal abduction and the tip of the probe placed perpendicular to the skin over the right lobe of the liver through the intercostal spaces [14]. The CAP was expressed as $\mathrm{dB} / \mathrm{m}$ and categorized into four stages: (1) steatosis stage 0 (S0) $<238 \mathrm{~dB} / \mathrm{m}$; (2) 238 $\mathrm{dB} / \mathrm{m} \leq$ steatosis stage $1(\mathrm{~S} 1)<260 \mathrm{~dB} / \mathrm{m}$; (3) $260 \mathrm{~dB} / \mathrm{m} \leq$ steatosis stage 2 (S2) $<293 \mathrm{~dB} / \mathrm{m}$; and (4) steatosis stage 3 (S3) $\geq$ $293 \mathrm{~dB} / \mathrm{m}$ [12]. In this study, the presence of NAFLD was defined as a CAP of $\geq 238 \mathrm{~dB} / \mathrm{m}$, i.e., steatosis stage 1 (S1) or greater than S1. Liver stiffness measurement (LSM) was assessed simultaneously and presented in kilopascals $(\mathrm{kPa})$. The stages of fibrosis were classified as (1) no or mild fibrosis ( $\mathrm{F}$ 0-1), <7.7 kPa; (2) significant fibrosis (F2), $\geq 7.7 \mathrm{kPa}$; (3) advanced fibrosis (F3), $\geq 8.5 \mathrm{kPa}$; and (4) cirrhosis (F4), $\geq 10.5$ $\mathrm{kPa}$ [15]. To ensure validity, at least 10 valid measurements were obtained for CAP/LSM, with interquartile ranges (IQRs) of CAP of $<40 \mathrm{~dB} / \mathrm{m}$ [16] and an IQR to median value ratio (IQR/M) of $<30 \%$ and an LSM success rate of $>60 \%$ were considered reliable [17].

\section{Statistical analyses}

Continuous variables with normal and non-normal distributions are expressed as mean \pm standard deviation and median with $\mathrm{IQR}$, respectively. Categorical variables are presented as frequencies and percentages. Independent sample $t$ tests and Mann-Whitney tests were used to compare continuous variables, while chi-square tests were used to compare categorical variables. Univariate logistic regression analyses were used to identify the potential predictors of NAFLD, with covariates with $P<0.2$ selected as candidate predictors [18]. Multivariable logistic regression analyses with NAFLD as the endpoint were performed to develop a new model. The backward selection method was applied from the initial model until we created final models with statistically significant covariates. The Hosmer-Lemeshow goodness-of-fit test was used to evaluate the model's fitness. Areas under the receiver operating characteristic curves (AUROCs) with $95 \%$ confidence intervals (CIs) were used to assess the model's performance, and the Youden index was used to determine effective cutoff values. The probabilities acquired from the final models were multiplied by 100 to obtain the scores.

We then compared our new models with previously devel- 
oped NAFLD screening models using development datasets. The established screening methods included the fatty liver index [19] from an Italian cohort and the hepatic steatosis index [20] and Park's index [21] from Korean cohorts. The sensitivity, specificity, positive predictive value (PPV), negative predictive value (NPV), positive likelihood ratio $(\mathrm{LR}+)$, negative likelihood ratio (LR-), and area under the curve were calculated [10]. The Jonckheere-Terpstra test was used to determine the significance of the trend. Statistical analyses were performed using SPSS Statistics for Windows version 25.0 (IBM Corp., Armonk, NY, USA) and R version 4.0.3 (R Foundation for Statistical Computing, Vienna, Austria). Statistical significance was set at $P<0.05$.

\section{RESULTS}

\section{Baseline characteristics of the study population}

Table 1 summarizes the characteristics of the study population according to NAFLD status. A total of 698 patients (445 men, 253 women) with a mean age of $59.4 \pm 9.6$ years were enrolled in the study. Fatty liver determined using TE was present in $64.6 \%(n=451)$ of the total patients. The patients in the NAFLD group were younger than those in the non-NAFLD group $(P=$ 0.006). The percentage of males was higher than females in the NAFLD and non-NAFLD groups without a significant intergroup difference in sex distribution. The mean BMI, waist circumference, and systolic and diastolic blood pressures were

Table 1. Baseline Characteristics of the Study Population According NAFLD Status

\begin{tabular}{|c|c|c|c|c|}
\hline Characteristic & $\begin{array}{c}\text { Total } \\
(n=698)\end{array}$ & $\begin{array}{l}\text { Non-NAFLD group } \\
\quad(n=247,35.4 \%)\end{array}$ & $\begin{array}{l}\text { NAFLD group } \\
(n=451,64.6 \%)\end{array}$ & $P$ value \\
\hline Age, yr & $59.4 \pm 9.6$ & $60.8 \pm 8.7$ & $58.7 \pm 10.0$ & $0.006^{\mathrm{a}}$ \\
\hline Male sex & $445(63.8)$ & $158(64)$ & $287(63.6)$ & 0.931 \\
\hline BMI, $\mathrm{kg} / \mathrm{m}^{2}$ & $25.1 \pm 3.3$ & $23.2 \pm 2.8$ & $26.1 \pm 3.1$ & $<0.001^{\mathrm{a}}$ \\
\hline Waist circumference, $\mathrm{cm}$ & $85.9 \pm 9.6$ & $80.6 \pm 8.4$ & $88.9 \pm 8.9$ & $<0.001^{\mathrm{a}}$ \\
\hline $\mathrm{SBP}, \mathrm{mm} \mathrm{Hg}$ & $126.2 \pm 15$ & $122.7 \pm 15.3$ & $128.1 \pm 14.6$ & $<0.001^{\mathrm{a}}$ \\
\hline DBP, mm Hg & $79.8 \pm 10.3$ & $77.6 \pm 9.7$ & $80.9 \pm 10.4$ & $<0.001^{\mathrm{a}}$ \\
\hline Hypertension & $363(52)$ & $114(46.2)$ & $249(55.2)$ & $0.002^{\mathrm{a}}$ \\
\hline Dyslipidemia & $358(51.3)$ & $120(48.6)$ & $238(52.8)$ & 0.106 \\
\hline Regular exercise & $496(71.1)$ & $196(79.4)$ & $300(66.5)$ & $0.010^{\mathrm{a}}$ \\
\hline Current smoker & $113(16.2)$ & $33(13.4)$ & $80(17.7)$ & 0.105 \\
\hline Current drinker ${ }^{\mathrm{b}}$ & $306(43.8)$ & 115 (46.6) & $191(42.4)$ & 0.431 \\
\hline Fasting glucose, mg/dL & $133.7 \pm 39.2$ & $127.1 \pm 39.3$ & $137.3 \pm 38.8$ & $0.001^{\mathrm{a}}$ \\
\hline HbAlc, $\%$ & $7.1 \pm 1.4$ & $6.9 \pm 1.3$ & $7.2 \pm 1.4$ & $<0.001^{\mathrm{a}}$ \\
\hline Uric acid, $\mathrm{mg} / \mathrm{dL}$ & $5.2 \pm 1.4$ & $5 \pm 1.3$ & $5.3 \pm 1.4$ & 0.065 \\
\hline Total cholesterol, mg/dL & $174.6 \pm 40.2$ & $174.7 \pm 42.1$ & $174.6 \pm 39.3$ & 0.972 \\
\hline Triglyceride, mg/dL & $109.5(76-151)$ & $91(64-124)$ & $121(88-164.5)$ & $<0.001^{\mathrm{a}}$ \\
\hline HDL-C, mg/dL & $46(39-54)$ & $48(41-61)$ & $44.5(39-52)$ & $<0.001^{\mathrm{a}}$ \\
\hline $\mathrm{LDL}-\mathrm{C}, \mathrm{mg} / \mathrm{dL}$ & $105.6 \pm 36$ & $102.8 \pm 37.4$ & $107.2 \pm 35.2$ & 0.127 \\
\hline AST, IU/L & $29(23-37)$ & $26(22-34)$ & $30(24-40)$ & $<0.001^{\mathrm{a}}$ \\
\hline ALT, IU/L & $29.5(22-42.3)$ & $25(19-34)$ & $33(24-48)$ & $<0.001^{\mathrm{a}}$ \\
\hline GGT, IU/L & $28(19-45)$ & $21(16-35)$ & $31(22-51.8)$ & $<0.001^{\mathrm{a}}$ \\
\hline $\mathrm{CAP}, \mathrm{dB} / \mathrm{m}$ & $254(224-291)$ & $212(196-228)$ & $280(256-306)$ & $<0.001^{\mathrm{a}}$ \\
\hline $\mathrm{LSM}, \mathrm{kPa}$ & $4.3(3.5-4.9)$ & $3.7(3.3-4.4)$ & $4.4(3.8-5.2)$ & $<0.001^{\mathrm{a}}$ \\
\hline
\end{tabular}

Values are expressed as mean \pm standard deviation, number (\%), or median (interquartile range).

NAFLD, nonalcoholic fatty liver disease; BMI, body mass index; SBP, systolic blood pressure; DBP, diastolic blood pressure; HbA1c, glycated hemoglobin A1c; HDL-C, high-density lipoprotein cholesterol; LDL-C, low-density lipoprotein cholesterol; AST, aspartate aminotransferase; ALT, alanine aminotransferase; GGT, gamma-glutamyl transferase; CAP, controlled attenuation parameter; LSM, liver stiffness measurement.

${ }^{\text {a }}$ Statistically significant values $(P<0.05)$; ${ }^{\mathrm{b}}$ Current drinker is defined as a person who drinks at least once a week. However, we excluded patients with excessive alcohol intake with following criteria; $\geq 210 \mathrm{~g} /$ week for males and $\geq 140 \mathrm{~g} /$ week for females. 
higher in the NAFLD group than in the non-NAFLD group, indicating that patients with NAFLD were more likely to be obese and have hypertension (all $P<0.05$ ). Patients in the NAFLD group exercised less than those in the non-NAFLD group. Glycemic parameters, including fasting glucose and $\mathrm{HbAlc}$ levels, were significantly higher in the NAFLD group. In addition, triglyceride levels were higher and high-density lipoprotein cholesterol (HDL-C) levels were lower in the NAFLD group than in the non-NAFLD group (all $P<0.05$ ). Regarding liver-associated parameters, AST, ALT, gamma-glutamyl transferase $(\gamma-\mathrm{GT})$, and hepatic steatosis (CAP) and stiffness by TE in the NAFLD group were significantly higher than in the non-NAFLD group $(P<0.001)$.

\section{Development of non-laboratory and comprehensive models for NAFLD}

Potential predictors of NAFLD were analyzed using a simple logistic regression model. Variables were selected based on clinical judgment and reported risk factors for NAFLD [22] and statistical significance in the current study. Age, waist circumference, BMI, dyslipidemia, hypertension, physical activity, smoking status, fasting glucose, HbAlc, triglyceride, HDL-C, low-density lipoprotein cholesterol, ALT/AST ratio, and $\gamma$-GT were chosen as candidate predictors of NAFLD in the univariate logistic regression model $(P<0.2)$ (Supplemental Table S1). We also included sex among the candidate predictors according to the clinical judgment [23]. Multivariable logistic regression analyses with backward selection method were performed to derive the final model. Table 2 presents the final non-laboratory and comprehensive model. Non-laboratory parameters, such as anthropometric data or variables obtained through inquiries, were used in the development of a simple non-laboratory model. This model comprised six variables, including age, sex, waist circumference, BMI, dyslipidemia, and smoking status, and fitted well ( $P=0.199$, Hosmer-Lemeshow statistics) with AUROCs of 0.780 (95\% CI, 0.743 to 0.817 ) (Fig. 1A). We multiplied the probabilities resulting from the non-laboratory model by 100 to obtain scores ranging from 0 to 100 , and the probability of having NAFLD was calculated as follows:

The non-laboratory model score, probability (\%) of having $\mathrm{NAFLD}=1 /(1+\exp (-\mathrm{x})) \times 100$.

$\mathrm{x}=-10.572+(-1.853) \times(1$ if age $\geq 40$ years, 0 if age $<40$ years $)+1.027 \times \operatorname{sex}($ female $=1$, male $=0)+0.112 \times$ waist $+0.12 \times$ $\mathrm{BMI}+0.227 \times$ dyslipidemia $(\mathrm{yes}=1, \mathrm{no}=0)+0.414 \times$ smoking status (current smoker $=1$, non-current smoker $=0$ )

These scores can be interpreted as the average probability of

\begin{tabular}{|c|c|c|}
\hline \multirow[b]{2}{*}{ Variable } & \multicolumn{2}{|c|}{ OR $(95 \% \mathrm{CI})$} \\
\hline & $\begin{array}{c}\text { Non-Laboratory } \\
\text { model }\end{array}$ & $\begin{array}{c}\text { Comprehensive } \\
\text { model }\end{array}$ \\
\hline Age, yr & - & $0.982(0.960-1.004)$ \\
\hline$<40$ & 1.0 (reference) & - \\
\hline$\geq 40$ & $0.157(0.018-1.357)$ & - \\
\hline \multicolumn{3}{|l|}{ Sex } \\
\hline Male & 1.0 (reference) & 1.0 (reference) \\
\hline Female & $2.791(1.715-4.544)$ & $3.370(2.070-5.486)$ \\
\hline Waist circumference, $\mathrm{cm}$ & $1.118(1.069-1.170)$ & $1.115(1.062-1.169)$ \\
\hline BMI, $\mathrm{kg} / \mathrm{m}^{2}$ & $1.128(0.996-1.277)$ & $1.122(0.986-1.276)$ \\
\hline Dyslipidemia & $1.255(0.868-1.814)$ & - \\
\hline Current smoking & $1.513(0.905-2.530)$ & - \\
\hline $\mathrm{HbAlc}, \%$ & - & $1.120(0.967-1.297)$ \\
\hline $\mathrm{TG}, \mathrm{mg} / \mathrm{dL}$ & - & $1.005(1.001-1.008)$ \\
\hline ALT/AST ratio & - & $4.342(2.218-8.501)$ \\
\hline AUC & 0.780 & 0.815 \\
\hline Nagelkerke $R^{2}$ & 0.304 & 0.381 \\
\hline \multicolumn{3}{|c|}{$\begin{array}{l}\text { Multivariable logistic regression analysis was performed. } \\
\text { The screening index of non-laboratory model }=1 /(1+\exp (-\mathrm{x})) \times 100 \text {; } \\
\mathrm{x}=-10.572+(-1.853) \times(1 \text { if age } \geq 40,0 \text { if age }<40)+1.027 \times \text { sex }(\mathrm{fe}- \\
\text { male }=1 \text {, male }=0)+0.112 \times \text { waist }+0.12 \times \mathrm{BMI}+0.227 \times \text { dyslipidemia } \\
(\mathrm{yes}=1 \text {, no }=0)+0.414 \times \text { smoking status }(\text { current smoker }=1 \text {, non-current } \\
\text { smoker }=0) \text {; The screening index of comprehensive model }=1 /(1+\mathrm{exp} \\
(-\mathrm{x})) \times 100 ; \mathrm{x}=-13.606+(-0.018) \times \text { age }+1.215 \times \mathrm{sex}(\mathrm{female}=1 \text {, } \\
\text { male }=0)+0.109 \times \text { waist }+0.115 \times \mathrm{BMI}+0.113 \times \mathrm{HbA} 1 \mathrm{c}+0.005 \times \mathrm{TG}+ \\
1.468 \times \mathrm{ALT} / \mathrm{AST} \text {. } \\
\text { NAFLD, nonalcoholic fatty liver disease; OR, odds ratio; CI, confi- } \\
\text { dence interval; BMI, body mass index; HbA1c, glycated hemoglobin } \\
\text { A1c; TG, triglyceride; ALT, alanine aminotransferase; AST, aspartate } \\
\text { aminotransferase; AUC, area under the curve. }\end{array}$} \\
\hline
\end{tabular}

\section{NAFLD in T2DM patients.}

Serum biomarkers were included in the development of the comprehensive model. Seven variables - age, sex, waist circumference, BMI, HbA1c, triglyceride, and ALT/AST ratiowere identified as significant predictors of NAFLD, and the comprehensive model also fitted well ( $P=0.243$, Hosmer-Lemeshow statistics). The comprehensive model showed an improved discrimination ability compared to the non-laboratory model, with an AUROC of 0.815 (95\% CI, 0.782 to 0.847) (Fig. 1B). We also multiplied the probabilities resulting from the comprehensive model by 100 to obtain an index score ranging from 0 to 100 . The probability of having NAFLD in the comprehensive model is calculated as follows:

The comprehensive model score, probability (\%) of having 

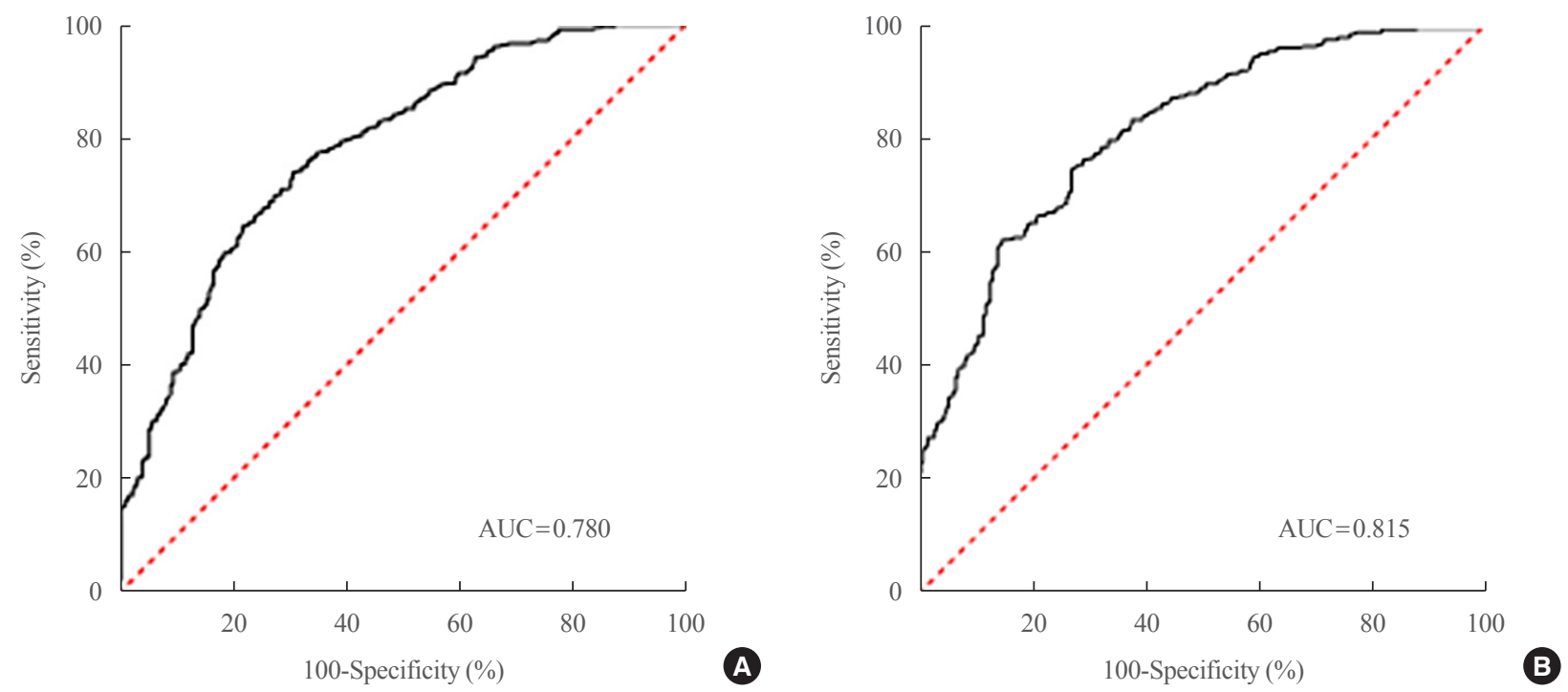

Fig. 1. (A) Receiver-operating characteristic (ROC) curve of non-laboratory screening model for the prediction of nonalcoholic fatty liver disease. The area under ROC curve (AUC) is 0.780 (95\% confidence interval [CI], 0.743 to 0.817 ). At cutoff value of 60 , sensitivity is $74 \%$, specificity is $69 \%$, respectively. (B) ROC curve of comprehensive screening model for the prediction of nonalcoholic fatty liver disease. The AUC is $0.815(95 \% \mathrm{CI}, 0.782$ to 0.847$)$. At cutoff value of 62 , sensitivity is $75 \%$, specificity is $73 \%$, respectively.

$\mathrm{NAFLD}=1 /(1+\exp (-\mathrm{x})) \times 100$.

$\mathrm{x}=-13.606+(-0.018) \times$ age $+1.215 \times$ sex $($ female $=1$, male $=$ $0)+0.109 \times$ waist $+0.115 \times \mathrm{BMI}+0.113 \times \mathrm{HbA1}+0.005 \times$ triglyceride $+1.468 \times$ ALT/AST

\section{Comparison with existing NAFLD screening models}

We compared the predictive power of our new screening indices with those of previously developed models, such as the hepatic steatosis index [20], Park's index [21], and fatty liver index [19], using our development dataset (Table 3). In the non-laboratory model, an index score of $\geq 60$ was set as the cutoff value, as it provided the highest Youden index with a sensitivity of $74 \%$, specificity of $69 \%$, PPV of $80 \%$, and NPV of $61 \%$. The comprehensive model showed a high risk of developing NAFLD for a cutoff score of $\geq 62$, which also provided the maximal Youden index. The comprehensive model showed a sensitivity of $75 \%$ and specificity of $73 \%$ for an index score of $\geq 62$. The sensitivity, specificity, PPV, NPV, LR+, LR-, and AUROCs of previously established screening models were analyzed using our development dataset. Our simple non-laboratory model was not inferior to other established models, including laboratory parameters. Moreover, the comprehensive model showed a numerically higher predictability for NAFLD than previous prediction models.

Fig. 2 shows the prevalence of NAFLD in both sexes according to score categories based on non-laboratory and comprehensive screening scores. The prevalence of NAFLD increased with increasing screening scores. Fatty liver grades assessed by TE showed significant associations with the new screening scores in simple linear regression models $\left(R^{2}=0.249\right.$ and $R^{2}=$ 0.332 in the non-laboratory and comprehensive models, respectively, $P<0.001$ ) (Supplemental Table S2). The screening scores increased with steatosis grade, suggesting that the new scores reflected the degree of fatty liver (Supplemental Fig. S1).

\section{DISCUSSION}

The worldwide distribution and increasing prevalence of NAFLD parallels the frequencies of obesity and unhealthy lifestyles, including in Asian countries [1,24]. Sedentary lifestyles and the prevalence of obesity have been constantly increasing in recent decades in Korea, contributing to the high prevalence of NAFLD $[4,25,26]$. Despite the increasing high-risk population of NAFLD, more than $95 \%$ of people with suspected NAFLD are unaware that they have liver disease [27]. Thus, many patients with NAFLD lack proper medical treatment, leading to liver-related and overall mortality. Due to its close association with cardiometabolic diseases, cancers, and end-stage liver disease [3], NAFLD accounts for a large part of public health expenses [28]. The diagnosis and treatment of NAFLD may enhance public health and reduce public health costs.

T2DM is a major risk factor for NAFLD [2]. The incidence of liver cirrhosis and mortality related to liver disease was higher in 
Table 3. Performance of NAFLD Screening Scores: Development Dataset

\begin{tabular}{lccccccccc}
\hline & $\begin{array}{c}\text { High-risk, } \\
\%\end{array}$ & $\begin{array}{c}\text { Sensitivity, } \\
\%\end{array}$ & $\begin{array}{c}\text { Specificity, } \\
\%\end{array}$ & $\begin{array}{c}\text { PPV, } \\
\%\end{array}$ & $\begin{array}{c}\text { NPV, } \\
\%\end{array}$ & $\begin{array}{c}\text { Positive } \\
\text { LR }\end{array}$ & $\begin{array}{c}\text { Negative } \\
\text { LR }\end{array}$ & $\begin{array}{c}\text { Youden } \\
\text { index }\end{array}$ & $\begin{array}{c}\text { AUC } \\
\text { Development dataset }(n=698)\end{array}$ \\
$\begin{array}{l}\text { Non-laboratory model } \geq 60 \\
\text { Comprehensive model } \geq 62\end{array}$ & 58 & 74 & 69 & 80 & 61 & 2.41 & 0.37 & 43 & 0.780 \\
Internal validation $(n=698)$ & 58 & 75 & 73 & 83 & 62 & 2.77 & 0.34 & 48 & 0.815 \\
Hepatic steatosis index & 51 & 65 & 74 & 82 & 53 & 2.49 & 0.48 & 41 & 0.779 \\
Park's index & 56 & 71 & 69 & 80 & 57 & 2.26 & 0.43 & 39 & 0.761 \\
Fatty liver index & 19 & 27 & 95 & 90 & 42 & 5.13 & 0.77 & 44 & 0.777 \\
\hline
\end{tabular}

Hepatic steatosis index $(\mathrm{HSI})=8 \times$ ALT/AST ratio+BMI $(+2$, if diabetes; +2 , if female), cutoff value of HSI $>36$ for high-risk or $<30$ for no NAFLD; Park's index $=($ ALT/AST ratio $>1.5) \times 1+(\gamma$-glutamyl-transferase $>50) \times 1+($ triglycerides $>150) \times 1+(23 \leq$ BMI $<25) \times 2+(25 \leq$ BMI $) \times 3$, cutoff value of Park's index $\geq 3$; Fatty liver index $(\mathrm{FLI})=1 /(1+\exp (-\mathrm{x})) \times 100, \mathrm{x}=0.953 \times \log _{\mathrm{e}}(\mathrm{TG})+0.139 \times \mathrm{BMI}+0.718 \times \log _{\mathrm{e}}(\gamma$-glutamyl-transferase $)+0.053 \times$ WC-15.745, cutoff value of FLI $\geq 60$ for high-risk or $<30$ for no NAFLD.

NAFLD, nonalcoholic fatty liver disease; PPV, positive predictive value; NPV, negative predictive value; LR, likelihood ratio; AUC, area under the curve; ALT, alanine aminotransferase; AST, aspartate aminotransferase; BMI, body mass index; TG, triglyceride; WC, waist circumference.
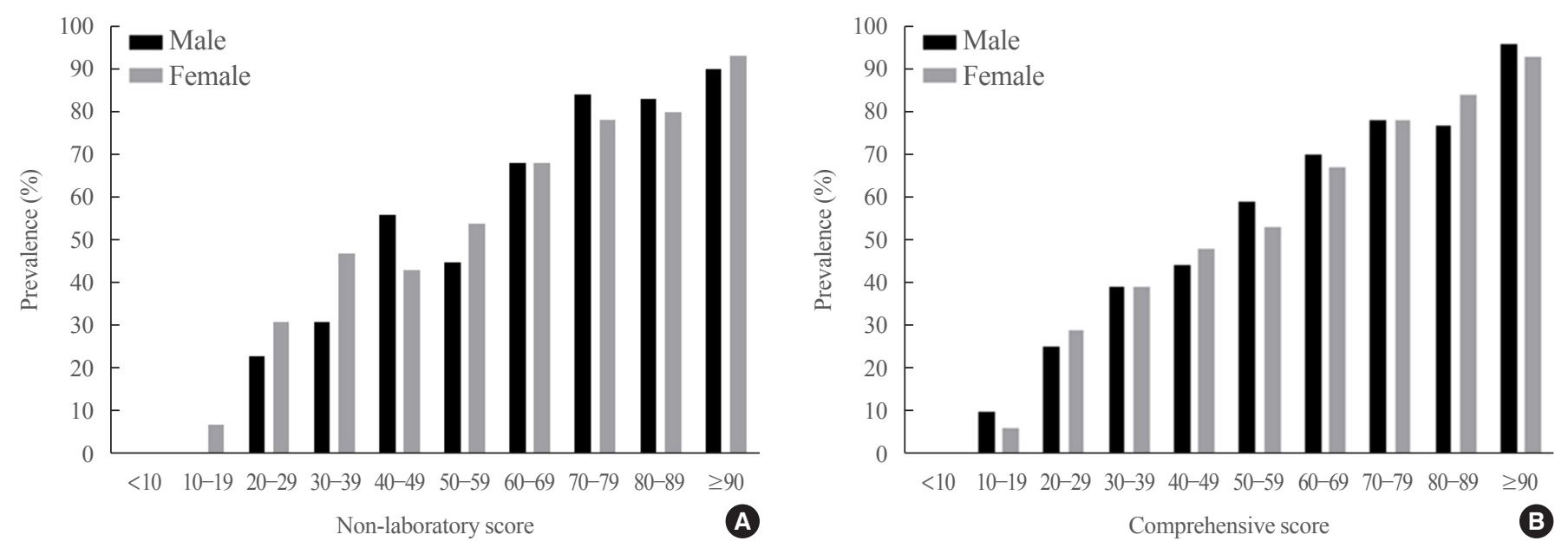

Fig. 2. Prevalence of nonalcoholic fatty liver according to score categories of screening models in development dataset. (A) Non-laboratory screening model and (B) comprehensive screening model.

patients with NAFLD and concomitant diabetes than in those with NAFLD but without diabetes [29]. The 2016 European Association for the Study of the Liver (EASL), European Association for the Study of Diabetes (EASD) and European Association for the Study of Obesity (EASO) Clinical Practice Guidelines for the management of NAFLD also recommend cautious examinations for the presence of NAFLD in patients with T2DM regardless of liver enzyme levels [3]. Thus, developing an effective modality for the early screening of NAFLD is especially crucial for patients with T2DM who have a higher risk and worse prognosis of NAFLD compared to the general population.

Various screening methods have been adopted to detect NAFLD, including ultrasonography, TE, CT, and MRI. Never- theless, there are many practical restrictions in the clinical setting in terms of the cost-effectiveness of performing imaging studies for NAFLD screening in all patients with T2DM. Therefore, we established non-laboratory and comprehensive models as a noninvasive and easy screening tool for NAFLD among patients with T2DM to identify individuals who require further evaluation and therapeutic interventions.

The results of the current study are consistent with those reported previously. NAFLD was highly prevalent in the present study, similar to previous observations [5]. Moreover, we also observed a close relationship between NAFLD and obesity other than alcohol consumption as reported previously $[22,30]$. In our prediction model, BMI and waist circumference, which rep- 
resent overall and central obesity, respectively, were important predictors of NAFLD and supported the tight association between fat accumulation and NAFLD [30]. Meanwhile, our models showed no association between the risk of NAFLD and alcohol consumption.

Our new prediction models differ from previous models in several ways. Most previous prediction models were developed for the general population and involved only the presence or absence of diabetes rather than the degree of glycemic control $[20,31,32]$. Our study confirmed the predictive power of the comprehensive model according to the degree of glycemic control in patients with T2DM. Our data indicate that clinicians should be aware of strict glycemic control to prevent the development of NAFLD. Unlike previously established NAFLD prediction models [10,31], our models do not show an increased risk of NAFLD with age. This finding is also supported by the study by Bedogni et al. [22] showing that NAFLD is not systematically associated with age. Younger patients in our development dataset tended to have a higher BMI and waist circumference, suggesting that younger adults were more obese (Supplemental Figs. S2, S3). According to the Korea National Health and Nutrition and Examination Survey (KNHANES) 2019, the prevalence of obesity was the highest among individuals in their $60 \mathrm{~s}(37.3 \%)$, followed by those in their $50 \mathrm{~s}(36.5 \%)$ and $40 \mathrm{~s}$ (35.6\%) [33], suggesting that more obese people are relatively younger. As fat accumulation is one of the major risk factors of NAFLD [2,3], this metabolic characteristic of young patients could explain the trend of the reverse relationship between age and NAFLD in the current prediction model. Our data also implied that fat accumulation may play a more important role than aging in the development of NAFLD.

There are conflicting views regarding the influence of sex on NAFLD. While some studies have described male predominance in NAFLD [24], others suggest a higher prevalence in women [34]. Other studies have not found an association between NAFLD and sex [22]. In the present study, female sex was identified as a risk factor for NAFLD. Like other chronic liver diseases, NAFLD is also influenced by the hormonal status. Although many studies demonstrated a higher prevalence of NAFLD in men than that in women, after menopause, the prevalence of NAFLD in women exceeded that of men [35-37]. Estrogen has protective effects against liver injury and reduce hepatic fibrosis progression in animal models [38]. While female sex hormones protect against hepatic steatosis in premenopausal women, this effect disappears after menopause. Unfavorable physiological changes occur in postmenopausal women, includ- ing fat redistribution and metabolic changes, such as increased insulin resistance, resulting in the development of NAFLD [39]. Unfortunately, information on menopausal status was not available for our dataset. However, considering that the mean age of the female participants in the study was $60.8 \pm 9.9$ years (Supplemental Table S3) and the mean age at natural menopause in Korean women is 49.3 years [40], most of the female participants in our dataset were presumed to be postmenopausal. Thus, the increased age with possible postmenopausal status in female patients might have affected the high odds ratio of NAFLD in women in the current study.

An increase in $\gamma$-GT is among the most common biochemical abnormalities in NAFLD, and $\gamma$-GT was included in the previous NAFLD prediction models [19,41]. However, $\gamma$-GT did not show statistical significance in our comprehensive model. The mitigated predictive power of $\gamma$-GT for NAFLD in the current study is probably due to the relatively early stages of NAFLD among the study population. Since $\gamma$-GT is an antioxidant that provides cellular defense in many cells, including hepatocytes [42], it is further increased with NAFLD progression [43]. According to a previous study [44], 23 of 102 individuals with normal liver findings on ultrasonography were diagnosed with NAFLD on TE, suggesting that TE is more sensitive than ultrasonography at detecting mild steatosis. Considering that previous NAFLD prediction models used ultrasonography [19], relatively advanced stages of NAFLD with sufficiently increased $\gamma$-GT levels may have been diagnosed, leading to $\gamma$-GT as a significant predictor of NAFLD. However, since the current study detected relatively early stages of hepatic steatosis using TE compared with previous studies using ultrasonography, $\gamma$-GT may not have increased enough to be a significant predictor of NAFLD. In addition, the median $\gamma$-GT value was $31 \mathrm{IU} / \mathrm{L}$, even in patients with NAFLD (Table 1), which was lower than the recommended cutoff value for the upper normal limit of $\gamma$-GT (51 IU/L for men and $33 \mathrm{IU} / \mathrm{L}$ for women) [45]. This implies that the current study mainly consisted of patients with early stages of NAFLD in which $\gamma$-GT had not been sufficiently increased to predict it. Furthermore, $\gamma$-GT can also be increased in other conditions of oxidative stress, such as alcohol-related diseases, heart failure, chronic kidney disease, dementia, and T2DM, along with increased insulin resistance [42,45]. The fact that the non-NAFLD control group was composed of only patients with T2DM might have resulted in the increased level of $\gamma$-GT in the non-NAFLD control group [46]. Therefore, the increased level of $\gamma$-GT in the non-NAFLD reference group may have reduced the difference in $\gamma$-GT levels between the NAFLD 
and non-NAFLD groups, decreasing the predictive power of $\gamma$-GT for NAFLD.

The present study has clinical significance owing to its novelty. First, pre-existing NAFLD prediction models were developed based on the diagnosis of NAFLD by ultrasonography or CT [10,19-21,31]. In contrast, we used TE as a diagnostic tool for NAFLD. Although ultrasonography is recommended as a first-line diagnostic imaging tool for NAFLD [3], TE is a promising device for simultaneously assessing liver fibrosis and steatosis $[12,15]$. For measuring CAP, TE is faster, more reproducible, and less operator-dependent than ultrasonography $[12,44]$. TE also shows improved diagnostic performance compared with CT [47]. Hence, we considered our prediction model as based on reliable methods for detecting NAFLD.

The second strength of the current study was that new predictive models were specifically developed in patients with T2DM. The pathophysiology of NAFLD in T2DM is distinct from that of NAFLD without diabetes. Glucotoxicity induced by persistent hyperglycemia with resultant chronic inflammation is the key pathophysiology of NAFLD in T2DM [48]. Therefore, an independent prediction model for NAFLD in T2DM patients is needed. Furthermore, to our knowledge, this is the first study to suggest a non-laboratory parameter-based prediction model of NAFLD determined using a FibroScan in patients with T2DM. The model is self-assessable with user-friendly parameters, and it enables high-risk patients with T2DM to easily calculate their probabilities of having NAFLD.

Our study has limitations that could be further investigated in subsequent studies. First, the levels of postprandial metabolic parameters such as glucose, insulin, and C-peptide were not measured due to the nature of routine healthcare checkups, although these parameters could be contributing factors for the prediction of NAFLD. For instance, a 1-hour postprandial glucose level above $155 \mathrm{mg} / \mathrm{dL}$ during an oral glucose tolerance test was associated with an increased risk of NAFLD and closely correlated with hepatic insulin resistance [49]. Hence, the inclusion of postprandial blood biomarkers in the NAFLD prediction model may reflect the components of hepatic insulin resistance in patients with T2DM. Therefore, further studies are needed to establish a more accurate prediction model of NAFLD that includes postprandial laboratory parameters. Second, although it is the gold standard for diagnosing NAFLD, liver biopsy was not performed in the present study. However, although TE is not a perfect method for diagnosing NAFLD, it could be considered a useful noninvasive substitute for liver biopsy considering its high diagnostic accuracy at detecting mild hepatic steatosis (sensitivity 87\%, specificity 91\%) [50]. Nevertheless, since there are some limitations of TE, further research is required to develop and validate NAFLD prediction models based on datasets from liver biopsy or MRI-based methods, which will provide excellent predictive power. Third, information on menopausal status that might affect the risk of NAFLD [39] could not be confirmed in the present study. Fourth, as external validation was not performed in the current study, systemic validation studies are warranted before the new prediction models can be utilized in clinical practice.

In conclusion, we developed and suggested new screening methods for NAFLD specifically for use in patients with T2DM at higher risk of developing NAFLD than the general population. The non-laboratory simple and comprehensive models were not inferior compared to existing models, and further investigations are needed to verify the efficacy and feasibility of these models in clinical practice. We hope that our new models will serve as noninvasive and cost-effective methods of screening for NAFLD in patients with T2DM.

\section{CONFLICTS OF INTEREST}

No potential conflict of interest relevant to this article was reported.

\section{ACKNOWLEDGMENTS}

This study was supported by the 'SENTINEL (Severance ENdocrinology daTa scIeNcE pLatform)' program funded by the 2020 Research fund of Department of Internal Medicine, Severance Hospital, Seoul, Korea and Sung-Kil Lim Research Award (4-2018-1215; DUCD000002) in statistical analyses. This work was supported by the Korean Endocrine Society of EnM Research Award 2018.

\section{AUTHOR CONTRIBUTIONS}

Conception or design: J.Y.N., E.S.K. Acquisition, analysis, or interpretation of data: J.K., M.L., S.Y.K., J.H.K., J.S.N., S.W.C., S.E.P., J.Y.N. Drafting the work or revising: J.K., M.L. Final approval of the manuscript: J.S.N., S.W.C., S.E.P., K.J.K., Y.L., J.Y.N., E.S.K.

\section{ORCID}

Jiwon Kim https://orcid.org/0000-0002-8390-4587 
Minyoung Lee https://orcid.org/0000-0002-9333-7512

Joo Young Nam https://orcid.org/0000-0003-1475-7285

Eun Seok Kang https://orcid.org/0000-0002-0364-4675

\section{REFERENCES}

1. Younossi ZM, Koenig AB, Abdelatif D, Fazel Y, Henry L, Wymer M. Global epidemiology of nonalcoholic fatty liver disease: meta-analytic assessment of prevalence, incidence, and outcomes. Hepatology 2016;64:73-84.

2. Chalasani N, Younossi Z, Lavine JE, Charlton M, Cusi K, Rinella $\mathrm{M}$, et al. The diagnosis and management of nonalcoholic fatty liver disease: practice guidance from the American Association for the Study of Liver Diseases. Hepatology 2018;67:328-57.

3. European Association for the Study of the Liver (EASL); European Association for the Study of Diabetes (EASD); European Association for the Study of Obesity (EASO). EASLEASD-EASO clinical practice guidelines for the management of non-alcoholic fatty liver disease. J Hepatol 2016;64: 1388-402.

4. Lee YH, Cho Y, Lee BW, Park CY, Lee DH, Cha BS, et al. Nonalcoholic fatty liver disease in diabetes. Part I: epidemiology and diagnosis. Diabetes Metab J 2019;43:31-45.

5. Younossi ZM, Golabi P, de Avila L, Paik JM, Srishord M, Fukui N, et al. The global epidemiology of NAFLD and NASH in patients with type 2 diabetes: a systematic review and meta-analysis. J Hepatol 2019;71:793-801.

6. Simeone JC, Bae JP, Hoogwerf BJ, Li Q, Haupt A, Ali AK, et al. Clinical course of nonalcoholic fatty liver disease: an assessment of severity, progression, and outcomes. Clin Epidemiol 2017;9:679-88.

7. Targher G, Bertolini L, Rodella S, Zoppini G, Lippi G, Day $\mathrm{C}$, et al. Non-alcoholic fatty liver disease is independently associated with an increased prevalence of chronic kidney disease and proliferative/laser-treated retinopathy in type 2 diabetic patients. Diabetologia 2008;51:444-50.

8. Lee JI, Kim MC, Moon BS, Song YS, Han EN, Lee HS, et al. The relationship between 10-year cardiovascular risk calculated using the pooled cohort equation and the severity of non-alcoholic fatty liver disease. Endocrinol Metab (Seoul) 2016;31:86-92.

9. Lee DH. Noninvasive evaluation of nonalcoholic fatty liver disease. Endocrinol Metab (Seoul) 2020;35:243-59.

10. Lee YH, Bang H, Park YM, Bae JC, Lee BW, Kang ES, et al. Non-laboratory-based self-assessment screening score for non-alcoholic fatty liver disease: development, validation and comparison with other scores. PLoS One 2014;9: e107584.

11. Kim MK, Ko SH, Kim BY, Kang ES, Noh J, Kim SK, et al. 2019 Clinical practice guidelines for type 2 diabetes mellitus in Korea. Diabetes Metab J 2019;43:398-406.

12. Sasso M, Beaugrand M, de Ledinghen V, Douvin C, Marcellin $\mathrm{P}$, Poupon R, et al. Controlled attenuation parameter (CAP): a novel VCTETM guided ultrasonic attenuation measurement for the evaluation of hepatic steatosis. Preliminary study and validation in a cohort of patients with chronic liver disease from various causes. Ultrasound Med Biol 2010; 36:1825-35.

13. Fraquelli M, Rigamonti C, Casazza G, Conte D, Donato MF, Ronchi G, et al. Reproducibility of transient elastography in the evaluation of liver fibrosis in patients with chronic liver disease. Gut 2007;56:968-73.

14. de Ledinghen V, Vergniol J. Transient elastography (FibroScan). Gastroenterol Clin Biol 2008;32(6 Suppl 1):58-67.

15. Gaia S, Carenzi S, Barilli AL, Bugianesi E, Smedile A, Brunello F, et al. Reliability of transient elastography for the detection of fibrosis in non-alcoholic fatty liver disease and chronic viral hepatitis. J Hepatol 2011;54:64-71.

16. Wong VW, Petta S, Hiriart JB, Camma C, Wong GL, Marra F, et al. Validity criteria for the diagnosis of fatty liver by $M$ probe-based controlled attenuation parameter. J Hepatol 2017;67:577-84.

17. Castera L, Forns X, Alberti A. Non-invasive evaluation of liver fibrosis using transient elastography. J Hepatol 2008; 48:835-47.

18. Maldonado G, Greenland S. Simulation study of confounder-selection strategies. Am J Epidemiol 1993;138:923-36.

19. Bedogni G, Bellentani S, Miglioli L, Masutti F, Passalacqua M, Castiglione A, et al. The Fatty Liver Index: a simple and accurate predictor of hepatic steatosis in the general population. BMC Gastroenterol 2006;6:33.

20. Lee JH, Kim D, Kim HJ, Lee CH, Yang JI, Kim W, et al. Hepatic steatosis index: a simple screening tool reflecting nonalcoholic fatty liver disease. Dig Liver Dis 2010;42:503-8.

21. Park YJ, Lim JH, Kwon ER, Kim HK, Jung MC, Seol KH, et al. Development and validation of a simple index system to predict nonalcoholic fatty liver disease. Korean J Hepatol 2011;17:19-26.

22. Bedogni G, Miglioli L, Masutti F, Tiribelli C, Marchesini G, Bellentani S. Prevalence of and risk factors for nonalcoholic fatty liver disease: the Dionysos nutrition and liver study. 
Hepatology 2005;42:44-52.

23. Sayiner M, Koenig A, Henry L, Younossi ZM. Epidemiology of nonalcoholic fatty liver disease and nonalcoholic steatohepatitis in the United States and the rest of the world. Clin Liver Dis 2016;20:205-14.

24. Farrell GC, Larter CZ. Nonalcoholic fatty liver disease: from steatosis to cirrhosis. Hepatology 2006;43(2 Suppl 1): S99-112.

25. Nam GE, Kim YH, Han K, Jung JH, Rhee EJ, Lee SS, et al. Obesity fact sheet in Korea, 2019: prevalence of obesity and abdominal obesity from 2009 to 2018 and social factors. J Obes Metab Syndr 2020;29:124-32.

26. Rhee EJ. Nonalcoholic fatty liver disease and diabetes: an epidemiological perspective. Endocrinol Metab (Seoul) 2019;34:226-33.

27. Singh A, Dhaliwal AS, Singh S, Kumar A, Lopez R, Gupta $\mathrm{M}$, et al. Awareness of nonalcoholic fatty liver disease is increasing but remains very low in a representative US cohort. Dig Dis Sci 2020;65:978-86.

28. Allen AM, Van Houten HK, Sangaralingham LR, Talwalkar JA, McCoy RG. Healthcare cost and utilization in nonalcoholic fatty liver disease: real-world data from a large U.S. claims database. Hepatology 2018;68:2230-8.

29. Younossi ZM, Gramlich T, Matteoni CA, Boparai N, McCullough AJ. Nonalcoholic fatty liver disease in patients with type 2 diabetes. Clin Gastroenterol Hepatol 2004;2:262-5.

30. Tilg H, Moschen AR, Roden M. NAFLD and diabetes mellitus. Nat Rev Gastroenterol Hepatol 2017;14:32-42.

31. Long MT, Pedley A, Colantonio LD, Massaro JM, Hoffmann U, Muntner P, et al. Development and validation of the framingham steatosis index to identify persons with hepatic steatosis. Clin Gastroenterol Hepatol 2016;14:1172-80.

32. Kotronen A, Peltonen M, Hakkarainen A, Sevastianova K, Bergholm R, Johansson LM, et al. Prediction of non-alcoholic fatty liver disease and liver fat using metabolic and genetic factors. Gastroenterology 2009;137:865-72.

33. Korea Centers for Disease Control and Prevention (KCDC). Korea National Health and Nutrition Examination Survey [Internet]. Cheongju: KCDC; 2019 [cited 2021 Jul 27]. Available from: http://www.kdca.go.kr/.

34. Ayonrinde OT, Olynyk JK, Beilin LJ, Mori TA, Pennell CE, de Klerk N, et al. Gender-specific differences in adipose distribution and adipocytokines influence adolescent nonalcoholic fatty liver disease. Hepatology 2011;53:800-9.

35. Park SH, Jeon WK, Kim SH, Kim HJ, Park DI, Cho YK, et al. Prevalence and risk factors of non-alcoholic fatty liver disease among Korean adults. J Gastroenterol Hepatol 2006; 21(1 Pt 1):138-43.

36. Zhou YJ, Li YY, Nie YQ, Ma JX, Lu LG, Shi SL, et al. Prevalence of fatty liver disease and its risk factors in the population of South China. World J Gastroenterol 2007;13: 6419-24.

37. Shen L, Fan JG, Shao Y, Zeng MD, Wang JR, Luo GH, et al. Prevalence of nonalcoholic fatty liver among administrative officers in Shanghai: an epidemiological survey. World J Gastroenterol 2003;9:1106-10.

38. Zhang H, Liu Y, Wang L, Li Z, Zhang H, Wu J, et al. Differential effects of estrogen/androgen on the prevention of nonalcoholic fatty liver disease in the male rat. J Lipid Res 2013; 54:345-57.

39. Suzuki A, Abdelmalek MF. Nonalcoholic fatty liver disease in women. Womens Health (Lond) 2009;5:191-203.

40. Park CY, Lim JY, Park HY. Age at natural menopause in Koreans: secular trends and influences thereon. Menopause 2018;25:423-9.

41. Armstrong MJ, Houlihan DD, Bentham L, Shaw JC, Cramb $\mathrm{R}$, Olliff S, et al. Presence and severity of non-alcoholic fatty liver disease in a large prospective primary care cohort. J Hepatol 2012;56:234-40.

42. Whitfield JB. Gamma glutamyl transferase. Crit Rev Clin Lab Sci 2001;38:263-355.

43. McPherson S, Hardy T, Henderson E, Burt AD, Day CP, Anstee QM. Evidence of NAFLD progression from steatosis to fibrosing-steatohepatitis using paired biopsies: implications for prognosis and clinical management. J Hepatol 2015;62:1148-55.

44. Yilmaz Y, Ergelen R, Akin H, Imeryuz N. Noninvasive detection of hepatic steatosis in patients without ultrasonographic evidence of fatty liver using the controlled attenuation parameter evaluated with transient elastography. Eur J Gastroenterol Hepatol 2013;25:1330-4.

45. Kunutsor SK. Gamma-glutamyltransferase: friend or foe within? Liver Int 2016;36:1723-34.

46. Sanyal D, Mukherjee P, Raychaudhuri M, Ghosh S, Mukherjee S, Chowdhury S. Profile of liver enzymes in non-alcoholic fatty liver disease in patients with impaired glucose tolerance and newly detected untreated type 2 diabetes. Indian J Endocrinol Metab 2015;19:597-601.

47. Zheng M, Chengliang C, Chen Y, Gopal N, Ramesh R, Jiao J. Diagnostic performance of fibroscan and computed tomography in 322 normal alanine aminotransferase nonobese non-alcoholic fatty liver disease patients diagnosed by 
ultrasound. Int J Clin Pract 2020;74:e13635.

48. Xian YX, Weng JP, Xu F. MAFLD vs. NAFLD: shared features and potential changes in epidemiology, pathophysiology, diagnosis, and pharmacotherapy. Chin Med J (Engl) 2020;134:8-19.

49. Sesti G, Hribal ML, Fiorentino TV, Sciacqua A, Perticone F. Elevated $1 \mathrm{~h}$ postload plasma glucose levels identify adults with normal glucose tolerance but increased risk of non-al- coholic fatty liver disease. BMJ Open Diabetes Res Care 2014;2:e000016

50. Pu K, Wang Y, Bai S, Wei H, Zhou Y, Fan J, et al. Diagnostic accuracy of controlled attenuation parameter (CAP) as a non-invasive test for steatosis in suspected non-alcoholic fatty liver disease: a systematic review and meta-analysis. BMC Gastroenterol 2019;19:51. 